\title{
ADVANCED CARBON FLUORIDES FOR PRIMARY LITHIUM BATTERIES
}

\author{
K. Guérin ${ }^{(1)(2)}$, M. Dubois ${ }^{(1)(2)}$, A. Hamwi ${ }^{(1)(2)}$ \\ (1) Université Clermont Auvergne, Université Blaise Pascal, Institut de Chimie de Clermont-Ferrand, BP 10448, F- \\ 63000 CLERMONT-FERRAND, France,Diane.Delbegue@univ-bpclermont.fr

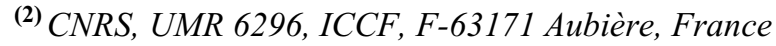

\begin{abstract}
Li-CFx battery using a specific fluorinated nanocarbon as cathode material exhibits a capacity exceeding the expected theoretical value when used as an electrode material in primary lithium battery. Carbon nanodiscs were partially fluorinated by atomic fluorine released by thermal decomposition of $\mathrm{TbF}_{4}$, and the capacity of this material was up to $1180 \mathrm{mAh} . \mathrm{g}^{-1}$, whereas a theoretical value of $847 \mathrm{mAh} . \mathrm{g}^{-1}$ for the $\mathrm{CF}_{0.95}$ sample was calculated. The obtained value is also higher than the maximum one of $865 \mathrm{mAh} . \mathrm{g}^{-1}$ expected for $\mathrm{CF}_{1}$ carbon fluorides. The discharge mechanism was investigated using mainly SEM and solid state NMR in order to understand this "extracapacity". Both the unfluorinated carbon and the $\mathrm{LiF}$ covering, which is formed outside the carbon lattice during the discharge mechanism, play a key role for the achievement of the extracapacity by the consumption of $\mathrm{Li}^{+}$to form $\mathrm{Li}_{2} \mathrm{~F}^{+}$species stabilized by the carbon host structure formed after the electrochemical defluorination.
\end{abstract}

\section{RESULTS AND DISCUSSION}

Carbon nanodiscs CND were fluorinated by atomic fluorine release by $\mathrm{TbF}_{4}$ decomposition at different temperatures. The fluorination progressively turns the structure as a function of the reaction temperature into a (CF) $n$ one. SEM images underlined the maintaining of the geometry for fluorination, whatever the reaction temperature. Nevertheless, as revealed by atomic force microscopy, the swelling due to the accommodation of the fluorine atoms differs according to the fluorination route as underlined by AFM, which was performed in tapping mode. The disc surface is smooth and cracks are observed on the whole surface. The accommodation of fluorine atoms in the sample is then homogeneous across discs thus leading to a homogeneous nonlocalized swelling. This therefore generates cracks on the material surface to accommodate the volume expansion associated with this process. Fluorinated CND were used as cathode material in primary lithium batteries. The two samples obtained by those fluorination way (C-500 and C-550 with atomic ratio $\mathrm{F} / \mathrm{C}=0.72$ and 0.95 , obtained at fluorination temperatures of 500 and $550^{\circ} \mathrm{C}$, respectively) were used as cathode in primary lithium batteries. Samples having $\boldsymbol{F} / \boldsymbol{C}$ of about $0.7-0.8$ were investigated because of its known good performances. [1, 2, 3].

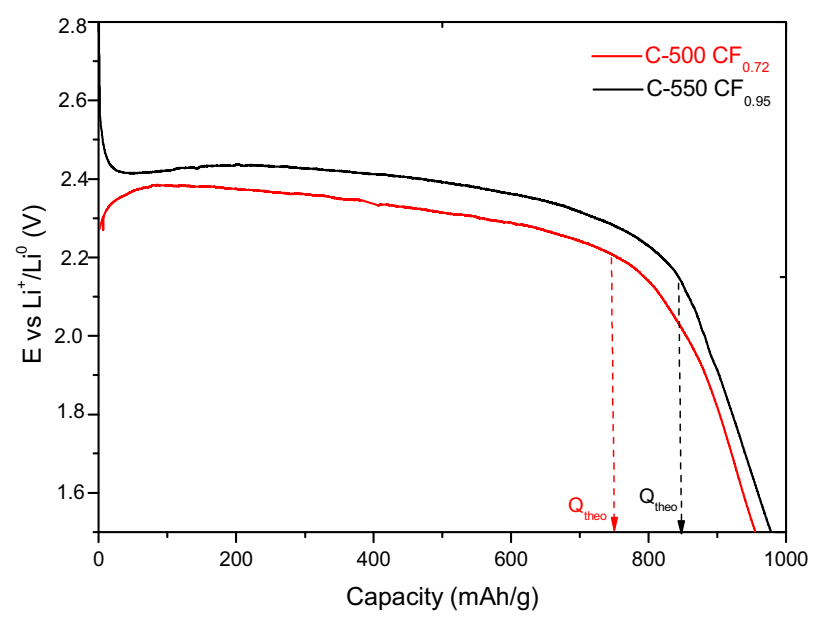

Figure 1. Galvanostatic discharge curves in EC/PC/3DMC LiPF6 $1 \mathrm{M}$ electrolyte at a current density of $10 \mathrm{~mA} . \mathrm{g}-1$ of fluorinated carbon nanodiscs (C-500 and C-550)

Figure 1 shows the galvanostatic discharge curves of $\mathrm{C}$ 500 and C-550 samples with EC/PC/3DMC LiPF6 electrolyte. Both samples exhibit the behavior usually expected for carbon fluorides with a well-defined plateau and initial overvoltage because of its insulating behavior. The voltage initially drops and then increases steadily to reach the main voltage plateau after few minutes; such a behavior has been already observed for graphite fluorides. $[4,5]$ The average potential is stable during the discharge in agreement with the unique C-F bonding (determined by ${ }^{13} \mathrm{C}$ and ${ }^{19} \mathrm{~F}$ solid state NMR), which is still covalent whatever the fluorination conditions. The most unexpected observation is that the experimental capacity (Qexp) significantly exceeds the theoretical value (Qtheo), for those two materials. Indeed, for C-550, the theoretical capacity is 847 mAh.g-1 while an experimental capacity of $978 \mathrm{mAh} . \mathrm{g}^{-1}$ is measured. This is also the case for C-500 sample, where $955 \mathrm{mAh} \cdot \mathrm{g}^{-1}$ as experimental capacity is measured instead of $751 \mathrm{mAh} \cdot \mathrm{g}^{-1}$. 
Different depths of discharge DoD, $0 \%, 25 \%, 50 \%$, $100 \%$ (relative to the theoretical capacity extracted from the fluorine content) and $127 \%$ (corresponding to the extracapacity), have been applied for a primary lithium battery with $\mathrm{C}-500$ as cathode in EC/PC/3DMC LiPF6 $1 \mathrm{M}$. After the discharge, the cathode material was dried under dynamic primary vacuum, transferred and ground in the argon-filled glove box and put into a rotor that allows NMR spectra to be recorded (without exposure to air) in order to investigate the discharge using ${ }^{19} \mathrm{~F}$ nucleus. After the recording of the NMR spectra, the electrode was investigated by EPR and SEM also without exposition to air.

SEM images of the C-500 composite electrode before and after discharges are shown in Figure 2. The good dispersion of PVDF binder is well highlighted for the raw electrode composite. The disc surface is covered with a film made with binder fibril (Figure 2a). In accordance with the well-known mechanism of formation of $\mathrm{LiF}$ with the reduction of $\mathrm{CFx}[1,6,7]$, these particles can be attributed to $\mathrm{LiF}$. The high concentration on the surface and edges suggests that $\mathrm{LiF}$ is formed outside the carbon lattice during the discharge. By analogy with lithium-graphite intercalation compounds, such as $\mathrm{LiC}_{6}, \mathrm{~F}^{-}$ions, which are formed during the fluorocarbon reduction, diffuse within the interlayer and combine with solvated $\mathrm{Li}+$ on the sheet edges. [8,9] A recent study on the discharge mechanism of fluorinated carbon nanofibres showed that such particles made of $\mathrm{LiF}$, are formed outside the carbonaceous matrix. [1] After the full theoretical discharge $(100 \%$ of faradic yield), the fluorocarbon matrix is totally converted into carbon. The peak related to the $(\mathrm{CF}) \mathrm{n}$ phase disappeared. No diffraction peak of ordered carbon lattice is observed meaning that amorphous carbon is formed. LiF peaks are observed at 39,45 and $65^{\circ}$ in $2 \boldsymbol{\theta}$ values. The formation of crystalline $\mathrm{LiF}$ particles is underlined by the sharpness of the peaks. It is to note that the discharge mechanism is nearly similar regardless the nanocarbon used, discs of fibres. [1] The LiF covering becomes thicker with increasing the discharge rate (DoD) up to $100 \%$.

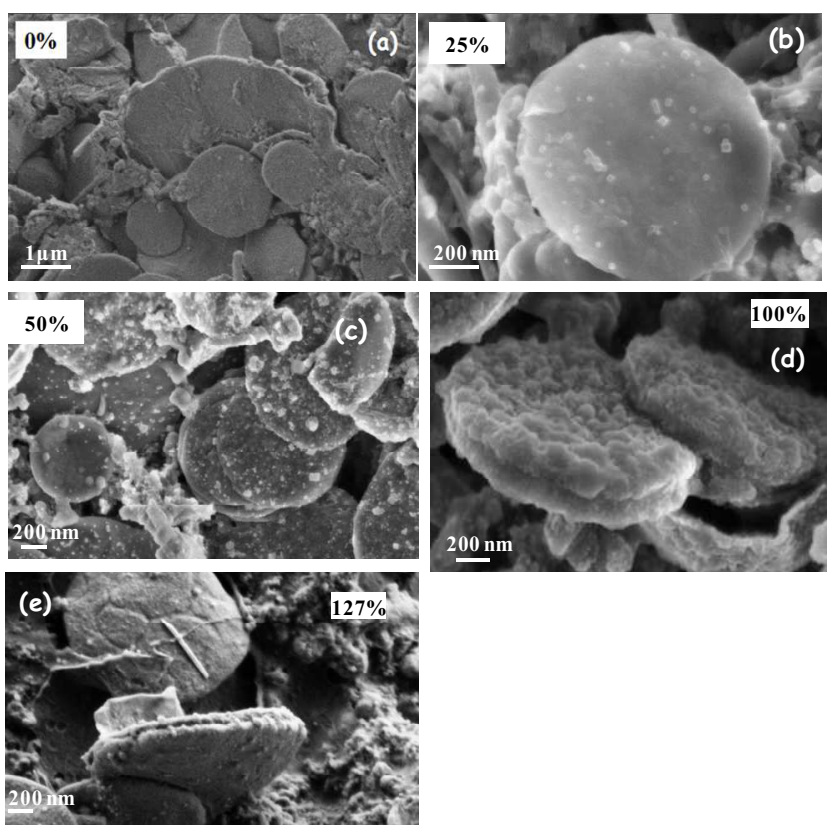

Figure 2. SEM images of C-500 at 0 (a), 25 (b), 50 (c) and $100 \%$ discharge ( $d$ and e). Figure (e) shows the sample with extracapacity (DoD of $127 \%$ )

After half of the discharge, discs and cones are still observed and are covered by particles with 20-30 nm size. The amount of these particles is higher on the cone and discs edges.

Finally, when an extracapacity (DoD of 127\%) is measured, the surface of the discs are covered by cracks that recall the disruptions observed on pristine fluorinated discs, well underlined by AFM images. Moreover, the discs seem to be split into two parts starting from their center.

Such data are in complete agreement with 19F MAS NMR, recorded at high spinning rate $(34 \mathrm{kHz})$ (Figure 3 ). The PVDF used as a binder is characterized by the presence of a line at $-94 \mathrm{ppm}$ assigned to $\mathrm{CF}_{2}$ groups. The amount of $\mathrm{CF}_{2}$ groups in the fluorinated materials underlined by a chemical shift of $-120 \mathrm{ppm} / \mathrm{CFCl}_{3}$ on the ${ }^{19} \mathrm{~F}$ NMR spectra does not change upon discharge. These groups are electrochemically inactive. The lines near $-80 \mathrm{ppm}$ are related to phosphorous species. The spectrum of solid $\mathrm{LiPF}_{6}$ is added for comparison and exhibits a line at $-74 \mathrm{ppm}$.

The pristine fluorinated CND sample contains small amount of $\mathrm{CF}_{2}$ groups, the lines for these groups being present with a very low intensity. As for the carbon 
nanofibres, the decrease of the intensity of the C-F line at $-190 \mathrm{ppm}$ as a function of the depth of discharge, is in accordance with the electrochemical breaking of the $\mathrm{C}-\mathrm{F}$ bonds during the discharge and it is accompanied by the formation of LiF. [1] For DoD of $100 \%$, this line is no more present underlining the total conversion of the fluorocarbon matrix into carbon lattice. The line at $204 \mathrm{ppm}$ is unambiguously assigned to the fluorine nuclei present in the particles of $\mathrm{LiF}$. The two lines with a chemical shift at -153 and -147 ppm are observed for discharged fluorinated CND with DoD of $127 \%$ unlike fluorinated CND discharged at DoD of 0 and $100 \%$ (Figure 3). A fluorinated specie, which is $\mathrm{Li} 2 \mathrm{~F}+\mathrm{is}$ obtained. Thus, our hypothesis to explain unprecedented extracapacity involves an insertion phenomenon of lithiated species Li2F+ into the carbon newly formed by electrochemical defluorination. This additional process could be the origin of the obtained extracapacities.

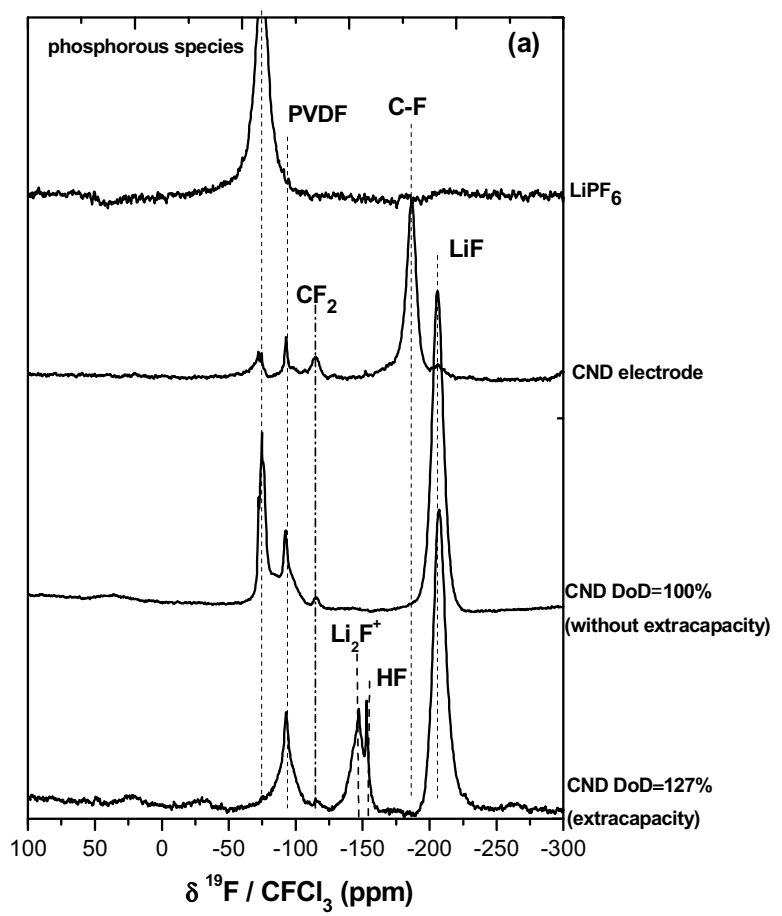

Figure 3. (a)19F MAS NMR spectra (34 kHz) of C500/PVDF/CB (80/10/10 w/w) composite electrode before discharge (composite with simple contact with the electrolyte), and after discharge with and without extracapacity (depths of discharge DoD $=100$ and $127 \%$ respectively). Lines at $-74 \mathrm{ppm}$ are related to phosphorous species as shown by the comparison with $\mathrm{LiPF}_{6}$ powder
Taking into account structural (NMR and EPR) and morphological (SEM and AFM) characterizations, the discharge mechanism, quasi-independent of the electrolyte may be described as follows. The discharge occurs successively according to the following steps: i) the growth of LiF particles because the breaking of C-F bonds and the recombination with $\mathrm{Li}+$ ions to form a continuous shell on the surface of the discs at $100 \%$ of discharge ii) carbon lattice is rebuilt onto the central disc, which serves as a reinforcement, iii) to exceed the theoretical capacity, an insertion process into the reformed carbon lattice may occur; the access of the ions into the carbon host takes place through the edges of discs but also via cracks on the initial fluorinated discs highlighted by AFM. The shell of LiF formed seems to have a major role in extracapacity; it seems to allow the diffusion of the lithium ions, either $\mathrm{Li}+$ or $\mathrm{Li}_{2} \mathrm{~F}^{+}$through the edges or the surface after the full discharge. As a matter of fact, the LiF shell seems to be locally consumed on the access areas for insertion. This mechanism is similar to that of the lithium-thionyl chloride batteries $\left(\mathrm{Li} / \mathrm{SOCl}_{2}\right)$ where the $\mathrm{LiCl}$ layer reacts with lithium ions to give $\mathrm{Li}_{2} \mathrm{Cl}^{+}$cations according to the reaction:

$$
\mathrm{Li}++\mathrm{LiCl} \rightarrow \mathrm{Li} 2 \mathrm{Cl}+
$$

which improves the performance of the cell. The splitting in two parts of the discs exactly on their center may be explained by two phenomena: either consumption of $\mathrm{LiF}$ as in the surface cracks or a volumic expansion because of the insertion.

The extracapacity may be obtained because of the maintaining of the geometry of the carbon lattice. This point must be explained taking into account the intrinsic properties of CND. Using freezing fracture, Naess et al [10] observed for the same nanodiscs from N-Tec an inplane cleavage that was usually parallel to the disc surface. The particles were fractured in a plane close to the geometrical centre of the disc. To establish this conclusion, a large number of TEM images (185) were analyzed. The histogram of the thickness ratio $\mathrm{x} 1 / \mathrm{x} 2(\mathrm{x} 1$ is the height of the lower part of the disc and $\mathrm{x} 2$ the height of the entire disc) shows that a significant number of discs rupture in an area close to the core. The splitting occurs between the graphitic layers, which are only bonded by weak van der Waals forces, and not in the amorphous carbon surrounding the disc. The authors concluded then the as-synthesized discs can be described as graphitic core within an envelope of noncrystalline carbon. The graphitic core is first grown to its full size, whereupon layers of amorphous carbon are deposited, the thickness of the graphitic core being estimated to only $2-5 \mathrm{~nm}$. Moreover, if the material is 
heat-treated at $2700^{\circ} \mathrm{C}$, the graphitization takes place simultaneously in different parts of the amorphous carbon. Thus the temperature-induced conversion from amorphous carbon to layers of graphite is not fully coupled geometrically to the original graphite core. Central discs are more organized than the outer discs after graphitization. The higher the graphitization, the lower the reactivity with fluorine. So the fluorination is higher in the outer parts than in the central discs, not or less fluorinated.

This history of the nanodiscs has important consequence during the fluorination/defluorination. An analogy between thermal and electrochemical defluorination is interesting. Ahmad et al. described the preparation of multilayer carbon nanomaterials obtained by thermal treatement (defluorination) of these sub-fluorinated nanocarbons obtained by the controlled fluorination process. [11] The defluorination occurs with a progressive thinning of the discs because of evolution of gaseous $\mathrm{CF}_{4}$ and $\mathrm{C}_{2} \mathrm{~F}_{6}$ species. Some carbons reorder onto the non or less fluorinated central discs resulting in very thin discs (thickness in the 5-20 nm range) which fold up on themselves. Homogeneous dispersion of fluorine atoms into the outer discs thanks to the controlled fluorination and lowering of the structural defects result in equivalent thinning in the whole discs. Any thinning occurs during the electrochemical defluorination but reduced carbon rebuilt onto the central discs, which act as a reinforcement to preserve the discotic geometry.

An insertion of lithiated ions may takes place into the complex structure of defluorinated carbon discs covered with $\mathrm{LiF}$. The $\mathrm{LiF}$ covering may acts both as lithium ions source and as diffusive layer for those ions. $\mathrm{LiF}$ is then probably consummed during the additional electrochemical process, assimilated as an insertion of lithiated ions $\mathrm{Li}+$ or $\mathrm{Li} 2 \mathrm{~F}+$ (in accordance with the additionnal line observed in $19 \mathrm{~F}$ and $7 \mathrm{Li}$ NMR spectra and charge transfer seen by EPR). LiF layer is then thinner at any zones of insertion, i.e. onto the sheet edges and onto the disruptions of carbon sheets, initially present in the raw fluorinated materials (well underlined by AFM).

Scheme 1 summarizes the electrochemical discharge mechanism and the insertion phenomenon which leads to extracapacity.

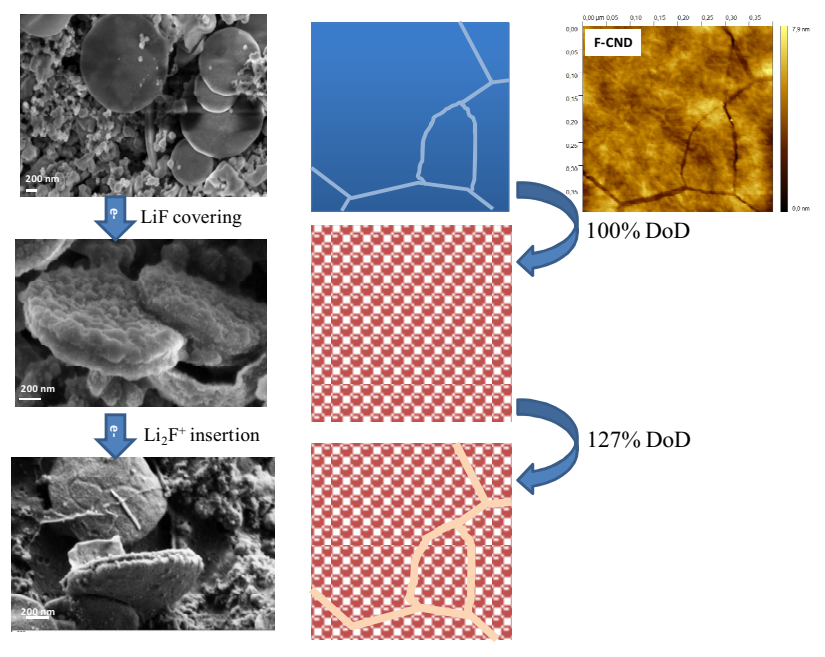

Scheme 1. Mechanisms explaining the extracapacity obtained with fluorinated CND

Since the $\mathrm{LiF}$ layer and the formation $\mathrm{Li}_{2} \mathrm{~F}^{+}$species play a major role in extracapacity, the thicker the LiF layer is, the greater the insertion of lithiated species and the extracapacity.

Taking into account that the ternary mixture of alkylcarbonates used as solvent for $\mathrm{EC} / \mathrm{PC} / 3 \mathrm{DMC} \mathrm{LiPF}_{6}$ $1 \mathrm{M}$ electrolyte is able to dissolve higher quantity of $\mathrm{LiF}$ $\left(\mathrm{SLiF}=9.08\right.$ g. $\mathrm{L}^{-1}$ ) compared to PC solvent (where $\mathrm{SLiF}=0.13$ g. $\mathrm{L}^{-1}$ ), extracapacity should be higher with

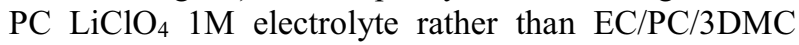
$\mathrm{LiPF}_{6} 1 \mathrm{M}$ electrolyte.

Thus, galvanostatic discharges at low current density (10 $\mathrm{mA} / \mathrm{g}$ ) were performed in $1 \mathrm{M} \mathrm{LiClO} 4 \mathrm{PC}$ electrolyte. A capacity up to $1180 \mathrm{mAh} / \mathrm{g}$ was observed, higher than $847 \mathrm{mAh} / \mathrm{g}$ expected for CF0.95 composition and enhanced in comparison with the one obtained in EC/PC/3DMC $\mathrm{LiPF}_{6} 1 \mathrm{M}$ electrolyte. Such experimental facts clearly underline that the extracapacity is related to the $\mathrm{LiF}$ covering and it can be improved via the choice of the electrolyte to reach $140 \%$ faradic yield. 


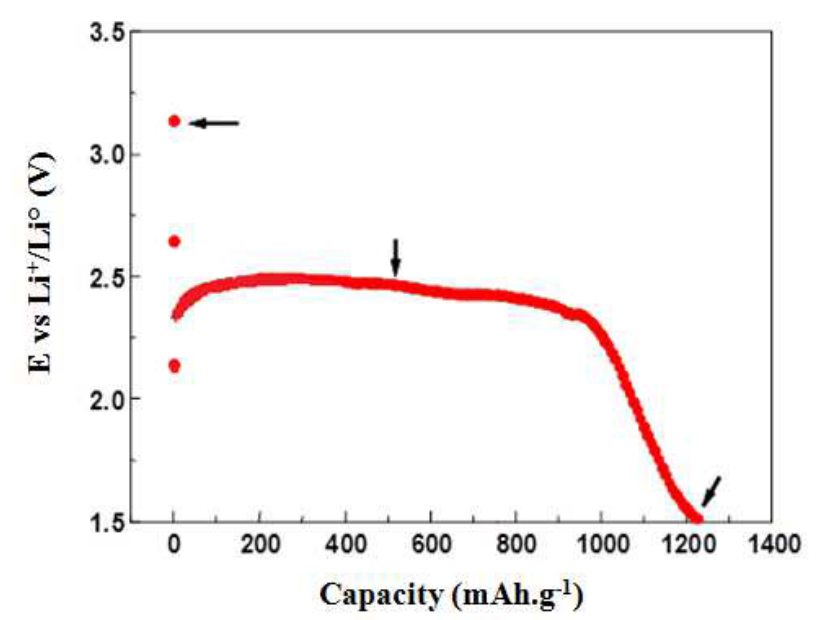

Figure 4. Galvanostatic discharge curve in $\mathrm{PC} \mathrm{LiClO} 4$ $1 \mathrm{M}$ electrolyte at a current density of $10 \mathrm{~mA} \cdot \mathrm{g}-1$ of CND fluorinated by controlled fluorination (C-550).

The extracapacity obtained there is the higher ever known in CFx primary lithium battery.

\section{References}

[1] Y. Ahmad, K. Guérin, M. Dubois, W. Zhang, A. Hamwi, Electrochim. Acta 2013, 114, 142.

[2] K. Guérin, M. Dubois, A. Houdayer, A. Hamwi, J. Fluorine Chem. 2012, 134, 11.

[3] R. Yazami, A. Hamwi, K. Guérin, Y. Ozawa, M. Dubois, J. Giraudet, F. Masin, Electrochem. Commun. 2007, 9, 1850.

[4] K. Guérin, R. Yazami, A. Hamwi, Electrochem. Solid-State Lett. 2004, 7, 159.

[5] M.J. Root, R. Dumas, R. Yazami, A. Hamwi, J. Electrochem. Soc. 2001, 148, 339.

[6] S. S. Zhang, D. Foster, J. Wolfenstine, J. Read, J. Power Sources 2009, 187, 233.

[7] E. Rangasamy, J. Li, G. Sahu, N. Dudney, C. Liang, J. Am. Chem. Soc. 2014, 136, 6874.

[8] J. Giraudet, M. Dubois, J. Inacio, A. Hamwi, Carbon 2003, 41, 453.

[9] A. Naji, P. Willmann, D. Billaud, Carbon 1998, 36, 1347.

[10] N. Stine Nalum, E. Arnljot, H. Geir, D. K. Kenneth, Sci. Technol. Adv. Mat. 2009, 10, 065002.

[11] Y. Ahmad, E. Disa, M. Dubois, K. Guérin, V. Dubois, W. Zhang, P. Bonnet, F. Masin, L. Vidal, D. A. Ivanov, A. Hamwi, Carbon 2012, 50, 3897. 